\title{
Estimating the compressibility of osmium from recent measurements of Ir-Os alloys under high pressure
}

\author{
N. V. Sarlis and E. S. Skordas, \\ Department of Solid State Physics and \\ Solid Earth Physics Institute \\ Faculty of Physics, School of Science, \\ National and Kapodistrian University of Athens, \\ Panepistimiopolis, Zografos 157 84, \\ Athens, Greece
}

August 1, 2018

\begin{abstract}
Several fcc- and hcp-structured Ir-Os alloys have been recently studied up to $30 \mathrm{GPa}$ at room temperature by means of synchrotron-based X-ray powder diffraction in diamond anvil cells. Using their bulk moduli -which increase with increasing osmium content showing a deviation from linearity- and after employing a thermodynamical model it was concluded that the bulk modulus for osmium is slightly smaller than that for diamond. Here, a similar conclusion is obtained upon employing an alternative model, thus strengthening the conclusion that osmium is the densest but not the most incompressible element. This is particularly interesting for Earth Sciences since it may be of key importance towards clarifying the anomalous elastic properties of the Earth's core.
\end{abstract}

\section{Introduction}

Heavy transition metals such as Ta, W, Re, Os and Ir have exceptional mechanical, thermal and chemical stabilities. Their very low compressibilities $\kappa$ make them essential subjects for high pressure studies. The compressibility of materials, in general, is of major importance because of its correlation to material strength, chemical bonding, and electronic structure. Strongly bonded materials have short interatomic distances and correspondingly strong repulsive forces, resulting in high bulk moduli $B(=1 / \kappa)$. In this regard, the finding by Cynn et al. (2002) that the $B$ value for Os at ambient conditions is $B=462(12) \mathrm{GPa}$, i.e., larger than that for diamond $(B=443 \mathrm{GPa}$, see Occelli et al. (2003)), attracted major interest. Such a large $B$ value has implications for the nature 
of the metallic bond in Os, where the bonding electrons are delocalized compared to the covalently bonded diamond in which they are localized. Later studies, however, came out with markedly lower $B$ values for Os. In particular, values around 400GPa were reported by Occelli et al. (2003), Kenichi (2004) and Godwal et al. (2012), while Voronin et al. (2005) found $B=435(19) \mathrm{GPa}$ and Armentrout and Kavner (2010) $B=421(3) \mathrm{GPa}$. Hence, controversial values concerning the bulk modulus for Os have been published to date. This is challenging in view of the following: Osmium is the densest element at ambient conditions, thus if its corresponding $B$ value exceeds that of diamond (as reported by Cynn et al. (2002)), this would reflect that Os is also the most incompressible element. In addition, osmium is of particular importance in Earth Sciences because it provides key information about the properties of iron and its alloys making up Earth's core: The stability of hcp osmium is similar to that of hcp iron, and a systematic characterization of these analog materials will help towards understanding the anomalous properties of Earth's core (Morelli et al., 1986; Creager, 1992). The melting point of osmium has been reported to be 3306K (Reiswig and Dickinson, 1964; Okamoto, 1994; Vacher et al., 1954; Geballe et al., 1963).

An interesting study of the compressibility of Ir-Os alloys under high pressure just appeared by Yusenko et al. (2015). As they noticed phase stability in the Ir-Os system has importance for the genesis of the natural Ir-Os-Ru and other pure platinum group based metallic minerals which show complex nature and non-equilibrium character at ambient conditions due to their formation under high-pressure from poly-component melt in Earth's mantle (Bird and Bassett, 1980; Fonseca et al., 2012; Weinberger et al., 2008). They prepared several fccand hcp- structured Ir-Os alloys and the measurement of the corresponding atomic volumes at ambient conditions using powder X-ray diffraction showed an almost linear dependence as a function of composition. These alloys were studied up to $P=30 \mathrm{GPa}$ at room temperature by means of synchrotron-based $\mathrm{X}$-ray powder diffraction in diamond anvil cells and their bulk moduli were found to increase with increasing osmium content showing a deviation from linearity. This concentration dependence of bulk moduli was shown by Yusenko et al. (2015) to be satisfactorily described by a thermodynamic model developed by Varotsos (1980a). According to this model the bulk moduli $B_{0}\left(x_{\mathrm{Os}}\right)$ as a function of the atomic fraction of osmium, $x_{\mathrm{Os}}$, is given by

$$
B_{0}\left(x_{\mathrm{Os}}\right)=B_{\mathrm{Ir}}\left[\frac{1+x_{\mathrm{Os}}\left(\frac{V_{\mathrm{Os}}}{V_{\mathrm{Ir}}}-1\right)}{1+x_{\mathrm{Os}}\left(\frac{B_{\mathrm{Ir}} V_{\mathrm{Os}}}{B_{\mathrm{Os}} V_{\mathrm{Ir}}}-1\right)}\right],
$$

where $B_{\mathrm{Ir}}$ and $B_{\mathrm{Os}}$ are bulk moduli and $V_{\mathrm{Ir}}$ and $V_{\mathrm{Os}}$ are atomic volumes at ambient pressure of pure Ir and Os, respectively. Yusenko et al. (2015), upon using their measured bulk moduli of the Ir-Os alloys and employing Eq.(1), found the values 354(2) GPa and 442(4)GPa for pure Ir and Os, respectively. In particular, Yusenko et al. (2015) concluded that the bulk modulus for pure Os is slightly smaller than that of the diamond. It is the scope of the present short 
paper to investigate the validity of this conclusion of Yusenko et al. (2015) by using an alternative model which interrelates the bulk modulus of an alloy with the bulk moduli of its pure constituents. The basic idea of this model is that when replacing an atom of a host crystal by a "foreign" atom, the corresponding volume variation can be considered as a "defect volume".

\section{The alternative model for the compressibility of a solid solution}

Let us explain how the compressibility $\kappa$ of a solid solution $\mathrm{A}_{x} \mathrm{~B}_{1-x}$ is interrelated with compressibilities of the two end members A and B by following Varotsos and Alexopoulos (1986) and Skordas (2012). We call the two end members $\mathrm{A}$ and $\mathrm{B}$ as pure components 1 and 2, respectively, and label $v_{1}$ the volume per atom of the pure component 1 and $v_{2}$ the volume per atom of the pure component 2. Let $V_{1}$ and $V_{2}$ denote the corresponding molar volumes, i.e. $V_{1}=N v_{1}$ and $V_{2}=N v_{2}$ (where $N$ stands for Avogadro's number) and assume that $v_{1}<v_{2}$. We now define a "defect volume" (Varotsos and Alexopoulos, 1986; Skordas, 2012) as the increase of the volume $V_{1}$, if one atom of type 1 is replaced by one atom of type 2 . It is evident that the addition of one "atom" of type 2 to a crystal containing atoms of type 1 will increase its volume by $v_{d}+v_{1}$ (see pp.325 and 326 of Varotsos and Alexopoulos (1986) as well as Skordas (2012)). Assuming that $v^{d}$ is independent of composition, the volume $V_{N+n}$ of a crystal containing $N$ atoms of type 1 and $n$ atoms of type 2 can be written as

$$
V_{N+n}=N v_{1}+n\left(v^{d}+v_{1}\right) \Longleftrightarrow V_{N+n}=\left[1+\frac{n}{N}\right] V_{1}+n v^{d}
$$

The molar fraction $x$ is connected to $n / N$ by (see Eq.(12.5) on page 328 of Varotsos and Alexopoulos (1986))

$$
\frac{n}{N}=\frac{x}{1-x}
$$

The compressibility $\kappa$ of the solid solution (as well as its bulk modulus $B=1 / \kappa$ ) can be found by differentiating Eq.(2) with respect to pressure, which finally gives:

$$
\kappa V_{N+n}=\kappa_{1} V_{1}+\frac{n}{N}\left[\kappa^{d} N v^{d}+\kappa_{1} V_{1}\right]
$$

where $\kappa^{d}$ denotes the compressibility of the volume $v^{d}$, defined as

$$
\kappa^{d} \equiv \frac{1}{B^{d}}=-\frac{1}{v^{d}}\left(\frac{\partial v^{d}}{\partial P}\right)_{T} .
$$

The "defect volume" $v^{d}$ can be approximated by (see p.342 of Varotsos and Alexopoulos (1986))

$$
v^{d}=\frac{V_{2}-V_{1}}{N}=v_{2}-v_{1} .
$$


Obviously $V_{N+n}$ can be obtained versus the composition by means of Eq.(2) when considering also Eq.(6). Then, the compressibility $\kappa$ can be studied versus the composition from Eq.(4) by assuming -to a first approximation- that the compressibility $\kappa^{d}$ is independent of composition. A rough estimation of $\kappa^{d}$ can be made by employing a thermodynamical model (Varotsos and Alexopoulos, 1977; Varotsos et al., 1978) for the formation and migration of the defects in solids (cf. the replacement of a host atom with a "foreign" one can be considered in general as a defect (Varotsos, 1974; Varotsos and Mourikis, 1974; Varotsos and Miliotis, 1974)). This model has been successfully applied to various categories of solids including diamond (Varotsos, 2007), oxides (Chroneos and Vovk, 2015a), semiconductors (Chroneos and Vovk, 2015b), silicates (Zhang and Shan, 2015) metals (Alexopoulos and Varotsos, 1981; Varotsos and Alexopoulos, 1980a), ionic crystals (Varotsos and Alexopoulos, 1980b), fluorides (Varotsos and Alexopoulos, 1981, 1980c; Varotsos et al., 1985; Varotsos, 2008), mixed alkali halides (Varotsos, 1980b, 1981; Varotsos and Alexopoulos, 1980d; Varotsos, 1978) as well as complex ionic materials under uniaxial stress that emit electric signals before fracture (Varotsos et al., 1999, 1992), thus explaining the signals detected before major earthquakes (Varotsos, 2006; Varotsos et al., 2009, 2008; Skordas et al., 2010; Sarlis et al., 2010). Within the frame of this thermodynamical model, which states that the defect Gibbs energy $g$ is proportional to the bulk modulus as well as to the mean volume per atom, we first obtain the defect volume $v\left[=(\partial g / \partial P)_{T}\right]$, and therefrom the compressibility $\kappa^{d}$ (see Eq.(8.31) in p.156 of Varotsos and Alexopoulos (1986)):

$$
\kappa^{d}=\frac{1}{B}-\frac{\left(\frac{\partial^{2} B}{\partial P^{2}}\right)_{T}}{\left(\frac{\partial B}{\partial P}\right)_{T}-1} .
$$

Since $\left(\frac{\partial^{2} B}{\partial P^{2}}\right)_{T}$ is negative (see p.157 of Varotsos and Alexopoulos (1986)), Eq.(7) indicates that $\kappa^{d}>\frac{1}{B}$, i.e., $B>B^{d}$.

\section{Application of the alternative model to Ir-Os alloys}

Equation (4) can be alternatively written as

$$
\left(\frac{V_{N+n}}{N+n}\right) \frac{1}{B}=\frac{V_{1}}{N} \frac{1}{B_{1}}+\frac{v^{d}}{B^{d}}\left(\frac{n}{N+n}\right)
$$

where $\frac{V_{N+n}}{N+n}$ for the alloys denotes the mean volume per atom given in Table 3 of Yusenko et al. (2015). This equation reveals that when plotting $\left(\frac{V_{N+n}}{N+n}\right) \frac{1}{B}$ versus $\frac{n}{N+n}(=x$, see Eq.(3)) and making a least square fitting to a straight line the slope leads to $\frac{v^{d}}{B^{d}}$-from which $B^{d}$ is calculated since $v^{d}$ may be approximated 


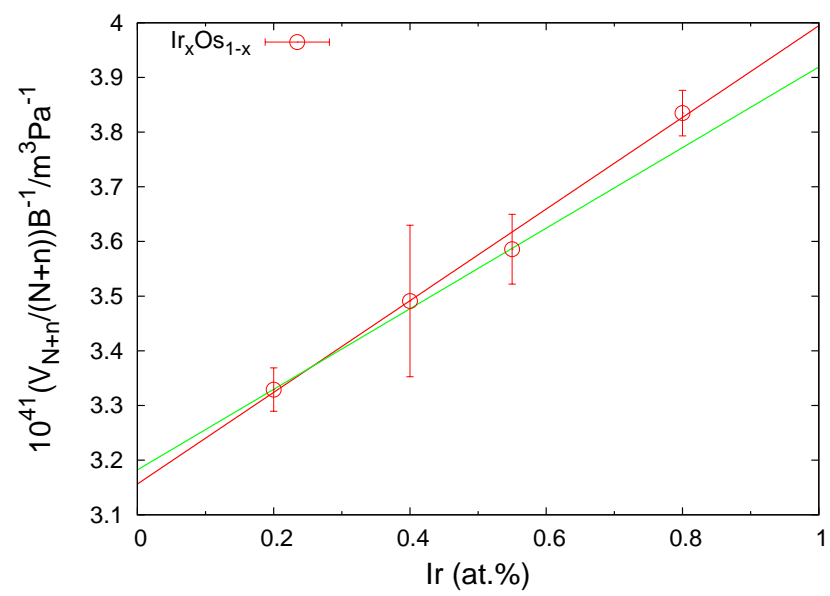

Figure 1: The composition dependence of the quantity $\left(\frac{V_{N+n}}{N+n}\right) \frac{1}{B}$ for the $\mathrm{Ir}_{x} \mathrm{Os}_{1-x}$ alloys studied by Yusenko et al. (2015). The red straight line corresponds to all four alloys (red circles) according to Eq.(8), while in the green straight line the highest Ir concentration $x=0.80$ is not considered (see the text).

by means of Eq.(6)- and the intercept results in $\frac{V_{1}}{N} \frac{1}{B_{1}}$ from which the bulk modulus $B_{1}$ of the pure component 1 is determined.

We follow the description of the previous Section and, for the sake of convenience, we assume as pure components 1 and 2 the elements Os and Ir (since the volume per atom in Os is smaller than that in Ir), thus we consider the alloys $\operatorname{Ir}_{x} \mathrm{Os}_{1-x}$. Yusenko et al. (2015) studied four compositions, i.e., $x=0.20,0.40,0.55$ and 0.80 , and in their Table 3 reported the following bulk moduli data $B=420(5), 403(16), 393(7)$, and 368(4)GPa, respectively. The mean volumes per atom for the two pure components are (Yusenko et al., 2015) $v_{1}=13.9825(1) \times 10^{-30} \mathrm{~m}^{3}$ and $v_{2}=14.1556(1) \times 10^{-30} \mathrm{~m}^{3}$ and hence $v^{d}=0.1731(1) \times 10^{-30} \mathrm{~m}^{3}$. Using these data, we now plot in Fig.1 with open red circles the values of $\left(\frac{V_{N+n}}{N+n}\right) \frac{1}{B}$ versus $\frac{n}{N+n}(=x)$ and make a weighted least squares fit (see pp. 659-660 of Press et al. (1992)).

The following results have been obtained: When employing all four alloys studied by Yusenko et al. (2015) we deduce the red straight line, the slope of which leads to $B^{d}=20.6(2.3) \mathrm{GPa}$ whereas its intercept to the bulk modulus of Os $B_{1}=443(8) \mathrm{GPa}$ and the corresponding ordinate for $x=1$ to the bulk modulus of $\mathrm{Ir}, B_{2}=354(10) \mathrm{GPa}$. If we do not consider the alloy with the highest Ir concentration, i.e., the one with $x=0.80$ (for which the concept of the "defects" introduced by Ir when considering Os matrix may not be fully justified) and restrict ourselves to the three alloys having the lower $x$ values, i.e., $x=0.20,0.40$, and 0.55 , we deduce the green straight line from which slightly different values are obtained. In particular, the slope leads to $B^{d}=$ 
23(7) GPa, its intercept to $B_{1}=439(10) \mathrm{GPa}$ and the value $x=1$ corresponds to $B_{2}=361(21) \mathrm{GPa}$. Thus, our results of the application of the alternative model to $\operatorname{Ir}_{x} \mathrm{Os}_{1-x}$ alloys could be summarized as follows: The bulk modulus $B_{1}$ for Os should lie between $439(10) \mathrm{GPa}$ and $443(8) \mathrm{GPa}$, while that $\left(B_{2}\right)$ of Ir between $354(10) \mathrm{GPa}$ and $361(21) \mathrm{GPa}$ (cf. as for the $B^{d}$ values they were found appreciably smaller than $B$ as expected from the thermodynamical model discussed in the previous Section). In other words, our findings concerning the bulk modulus of Os do not support the claim by Cynn et al. (2002) that it is markedly larger than the bulk modulus reported by Occelli et al. (2003) for diamond which is $443 \mathrm{GPa}$. As for our findings concerning the bulk modulus $B_{2}$ of Ir, we see that it lies between the values 354(6)GPa and 391(4)GPa reported by Cerenius and Dubrovinsky (2000) and Goncharov et al. (2007), respectively.

\section{Summary and Conclusions}

Here, we made use of the bulk moduli of fcc- and hcp-structured Ir-Os alloys recently measured by Yusenko et al. (2015) in order to estimate the bulk modulus of osmium for which controversial values have been reported. We find that it lies between 439(10)GPa and 443(8)GPa by employing a model different than that used in (Yusenko et al., 2015). Our results strengthen the conclusion that the bulk modulus for Os is slightly smaller in comparison with diamond ( $B=443 \mathrm{GPa})$. Furthermore, our findings contradict the earlier claim of Cynn et al. (2002) that osmium is less compressible than diamond, which is particularly interesting due to the significance of Os as a possible model of iron behavior in the Earth's core.

\section{Acknowledgments}

The experimental data used in the present paper come from Tables 2 and 3 of Yusenko et al. (2015).

\section{References}

Alexopoulos, K., and P. Varotsos (1981), Calculation of diffusion coefficients at any temperature and pressure from a single measurement. II. Heterodiffusion, Phys. Rev. B, 24, 3606-3609, doi:10.1103/PhysRevB.24.3606.

Armentrout, M. M., and A. Kavner (2010), Incompressibility of osmium metal at ultrahigh pressures and temperatures, J. Appl. Phys., 107(9), 093,528, doi:10.1063/1.3369283.

Bird, J. M., and W. A. Bassett (1980), Evidence of a deep mantle history in terrestrial osmium-iridium-ruthenium alloys, J. Geophys. Res.: Solid Earth, 85, 2156-2202, doi:10.1029/JB085iB10p05461. 
Cerenius, Y., and L. Dubrovinsky (2000), Compressibility measurements on iridium, Journal of Alloys and Compounds, 306(1-2), 26 - 29, doi:10.1016/S09258388(00)00767-2.

Chroneos, A., and R. Vovk (2015a), Modeling self-diffusion in $\mathrm{UO}_{2}$ and $\mathrm{ThO}_{2}$ by connecting point defect parameters with bulk properties, Solid State Ionics, 274, 1 - 3, doi:http://dx.doi.org/10.1016/j.ssi.2015.02.010.

Chroneos, A., and R. Vovk (2015b), Connecting bulk properties of germanium with the behavior of self- and dopant diffusion, $M a$ terials Science in Semiconductor Processing, 36, 179 - 183, doi: http://dx.doi.org/10.1016/j.mssp.2015.03.053.

Creager, K. C. (1992), Anisotropy of the inner core from differential travel times of the phases, Nature (London), 356, 309, doi:10.1038/356309a0.

Cynn, H., J. E. Klepeis, C.-S. Yoo, and D. A. Young (2002), Osmium has the lowest experimentally determined compressibility, Phys. Rev. Lett., 88, 135,701, doi:10.1103/PhysRevLett.88.135701.

Fonseca, R. O., V. Laurenz, G. Mallmann, A. Luguet, N. Hoehne, and K. P. Jochum (2012), New constraints on the genesis and long-term stability of Osrich alloys in the Earth's mantle, Geochim. Cosmochim. Acta, 87, $227-242$, doi:10.1016/j.gca.2012.04.002.

Geballe, T. H., B. T. Matthias, V. B. Compton, E. Corenzwit, and G. W. Hull (1963), Superconductivity of solid solutions of noble metals, Phys. Rev., 129, 182-183, doi:10.1103/PhysRev.129.182.

Godwal, B. K., J. Yan, S. M. Clark, and R. Jeanloz (2012), High-pressure behavior of osmium: An analog for iron in earth's core, J. Appl. Phys., 111(11), 112,608, doi:10.1063/1.4726203.

Goncharov, A. F., J. C. Crowhurst, J. K. Dewhurst, S. Sharma, C. Sanloup, E. Gregoryanz, N. Guignot, and M. Mezouar (2007), Thermal equation of state of cubic boron nitride: Implications for a high-temperature pressure scale, Phys. Rev. B, 75, 224,114, doi:10.1103/PhysRevB.75.224114.

Kenichi, T. (2004), Bulk modulus of osmium: High-pressure powder x-ray diffraction experiments under quasihydrostatic conditions, Phys. Rev. B, 70, 012,101, doi:10.1103/PhysRevB.70.012101.

Morelli, A., A. M. Dziewonski, and J. H. Woodhouse (1986), Anisotropy of the inner core inferred from pkikp travel times, Geophys. Res. Lett., 13, 1545, doi:10.1029/GL013i013p01545.

Occelli, F., P. Loubeyre, and R. LeToullec (2003), Properties of diamond under hydrostatic pressures up to $140 \mathrm{GPa}$, Nat. Mater., 2, 151-154, doi: $10.1038 /$ nmat831. 
Okamoto, H. (1994), The Ir-Os (iridium-osmium) system, J. Phase Equilib., 15, 55-57, doi:10.1007/BF02667683.

Press, W. H., S. Teukolsky, W. Vetterling, and B. P. Flannery (1992), Numerical Recipes in FORTRAN, 963 pp., Cambridge University Press, New York.

Reiswig, R. D., and J. M. Dickinson (1964), The osmium-iridium equilibrium diagram, Trans. Metall. Soc. AIME, 230, 469-472.

Sarlis, N. V., E. S. Skordas, and P. A. Varotsos (2010), Nonextensivity and natural time: The case of seismicity, Phys. Rev. E, 82, 021,110, doi: 10.1103/PhysRevE.82.021110.

Skordas, E. S. (2012), Comments on the elastic properties in solid solutions of silver halides, Mod. Phys. Lett. B, 26, 1250,066, doi: 10.1142/S0217984912500662.

Skordas, E. S., N. V. Sarlis, and P. A. Varotsos (2010), Effect of significant data loss on identifying electric signals that precede rupture estimated by detrended fluctuation analysis in natural time, CHAOS, 20, 033,111, doi: 10.1063/1.3479402.

Vacher, H. C., C. J. Bechtoldt, and E. Maxwell (1954), Structure of some osmium-iridium alloys, J. Metal., 200, 80-82.

Varotsos, P. (1974), Conductivity and dielectric constants of LiD, Phys. Rev. B, 9, 1866-1869, doi:10.1103/PhysRevB.9.1866.

Varotsos, P. (1978), An estimate of the pressure dependence of the dielectric constant in alkali halides, Phys. Stat. Sol. (b), 90, 339-343.

Varotsos, P. (1980a), On the temperature variation of the bulk modulus of mixed alkali halides, Phys. Status Sol. B, 99(2), K93-K96, doi: $10.1002 /$ pssb.2220990243.

Varotsos, P. (1980b), Determination of the dielectric constant of alkali halide mixed crystals, physica status solidi (b), 100(2), K133-K138, doi: $10.1002 /$ pssb.2221000252.

Varotsos, P. (1981), Determination of the composition of the maximum conductivity or diffusivity in mixed alkali halides, J. Phys. Chem. Sol., 42, 405-407, doi:10.1016/0022-3697(81)90048-2.

Varotsos, P. (2008), Point defect parameters in $\beta-\mathrm{PbF}_{2}$ revisited, Solid State Ionics, 179(11?12), 438 - 441, doi:10.1016/j.ssi.2008.02.055.

Varotsos, P., and K. Alexopoulos (1977), Calculation of the formation entropy of vacancies due to anharmonic effects, Phys. Rev. B, 15, 4111-4114, doi: 10.1103/PhysRevB.15.4111. 
Varotsos, P., and K. Alexopoulos (1980a), Determination of the Compressibility of an Alloy from Its Density, physica status solidi (b), 102(1), K67-K72, doi: $10.1002 /$ pssb.2221020157.

Varotsos, P., and K. Alexopoulos (1980b), On the question of the calculation of migration volumes in ionic crystals, Philosophical Magazine A, 42(1), 13-18, doi:10.1080/01418618008239352.

Varotsos, P., and K. Alexopoulos (1980c), Migration entropy for the bound fluorine motion in alkaline earth fluorides, J. Phys. Chem. Sol., 41, 443-446, doi:10.1016/0022-3697(80)90172-9.

Varotsos, P., and K. Alexopoulos (1980d), Prediction of the compressibility of mixed alkali halides, Journal of Physics and Chemistry of Solids, 41(12), 1291 $-1294$.

Varotsos, P., and K. Alexopoulos (1981), Migration parameters for the bound fluorine motion in alkaline earth fluorides. II, J. Phys. Chem. Sol., 42, $409-$ 410, doi:10.1016/0022-3697(81)90049-4.

Varotsos, P., and K. Alexopoulos (1986), Thermodynamics of Point Defects and their Relation with Bulk Properties, 474 pp., North Holland, Amsterdam.

Varotsos, P., and S. Miliotis (1974), New aspects on the dielectric properties of the alkali halides with divalent impurities, J. Phys. Chem. Sol., 35, 927-930.

Varotsos, P., and S. Mourikis (1974), Difference in conductivity between lid and lih crystals, Phys. Rev. B, 10, 5220-5224.

Varotsos, P., W. Ludwig, and K. Alexopoulos (1978), Calculation of the formation volume of vacancies in solids, Phys. Rev. B, 18, 2683-2691, doi: 10.1103/PhysRevB.18.2683.

Varotsos, P., K. Alexopoulos, C. Varotsos, and M. Lazaridou (1985), Interconnection of point defect parameters in $\mathrm{BaF}_{2}$, physica status solidi (a), 88(2), K137-K140, doi:10.1002/pssa.2210880250.

Varotsos, P., N. Bogris, and A. Kyritsis (1992), Comments on the depolarization currents stimulated by variations of temperature and pressure, J. Phys. Chem. Solids, 53, 1007-1011, doi:10.1016/0022-3697(92)90069-P.

Varotsos, P. A. (2006), What happened before the last five strong earthquakes in greece, Proc. Jpn. Acad., Ser. B: Phys. Biol. Sci., 82, 86-91, doi: 10.2183/pjab.82.86.

Varotsos, P. A. (2007), Calculation of point defect parameters in diamond, Phys. Rev. B, 75, 172,107, doi:10.1103/PhysRevB.75.172107. 
Varotsos, P. A., N. V. Sarlis, E. S. Skordas, and M. S. Lazaridou (2008), Fluctuations, under time reversal, of the natural time and the entropy distinguish similar looking electric signals of different dynamics, J. Appl. Phys., 103, 014906, doi:10.1063/1.2827363.

Varotsos, P. A., N. V. Sarlis, and E. S. Skordas (2009), Detrended fluctuation analysis of the magnetic and electric field variations that precede rupture, CHAOS, 19, 023,114, doi:10.1063/1.3130931.

Varotsos, P. V., N. V. Sarlis, and M. S. Lazaridou (1999), Interconnection of defect parameters and stress-induced electric signals in ionic crystals, Phys. Rev. B, 59, 24-27, doi:10.1103/PhysRevB.59.24.

Voronin, G., C. Pantea, T. Zerda, L. Wang, and Y. Zhao (2005), Thermal equation-of-state of osmium: a synchrotron x-ray diffraction study, Journal of Physics and Chemistry of Solids, 66(5), 706 - 710, doi: 10.1016/j.jpcs.2004.08.045.

Weinberger, M. B., S. H. Tolbert, and A. Kavner (2008), Osmium metal studied under high pressure and nonhydrostatic stress, Phys. Rev. Lett., 100, 045,506, doi:10.1103/PhysRevLett.100.045506.

Yusenko, K. V., E. Bykova, M. Bykov, S. A. Gromilov, A. V. Kurnosov, C. Prescher, V. B. Prakapenka, M. Hanfland, S. van Smaalen, S. Margadonna, and L. S. Dubrovinsky (2015), Compressibility of Ir-Os alloys under high pressure, J. Alloys Compd., 622, 155-161, doi:10.1016/j.jallcom.2014.09.210.

Zhang, B., and S. Shan (2015), Application of the cB $\Omega$ model to the calculation of diffusion parameters of $\mathrm{Si}$ in silicates, Geochemistry, Geophysics, Geosystems, 16(3), 705-718, doi:10.1002/2014GC005551. 